Document downloaded from:

http://hdl.handle.net/10251/140211

This paper must be cited as:

Bailo Ballarín, E.; Gelonch, J.; Romero Vivó, S. (10-2). A first cubic upper bound on the local reachability index for some positive 2-D systems. Revista de la Real Academia de Ciencias Exactas Físicas y Naturales Serie A Matemáticas. 113(4):3767-3784. https://doi.org/10.1007/s13398-019-00699-0

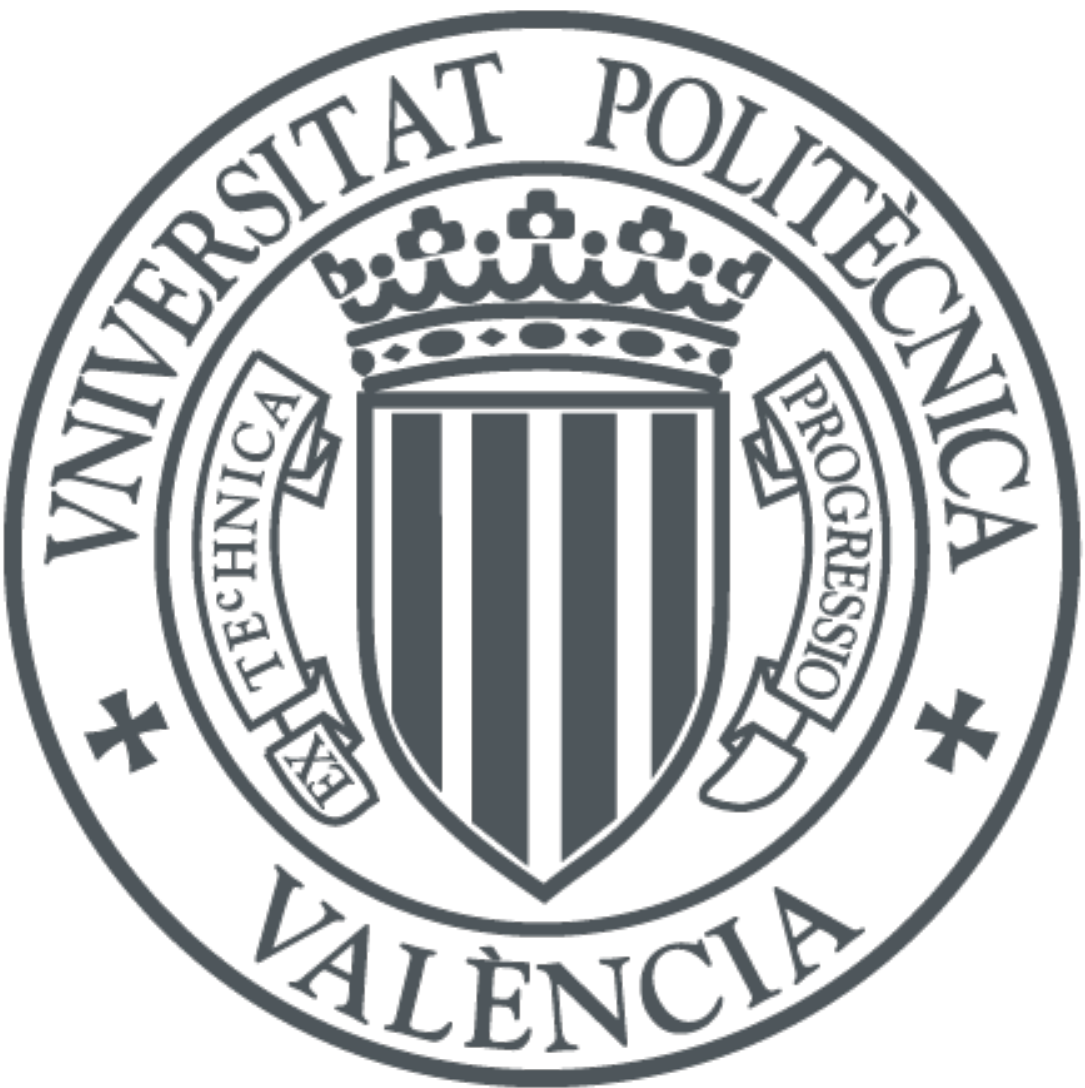

The final publication is available at

https://doi.org/10.1007/s13398-019-00699-0

Copyright Springer-Verlag

Additional Information 


\title{
A first cubic upper bound on the local reachability index for some positive 2-D systems
}

\author{
Esteban Bailo · Josep Gelonch · Sergio \\ Romero-Vivó
}

Received: date / Accepted: date

\begin{abstract}
The calculation of the smallest number of steps needed to deterministically reach all local states of an $n$ th-order positive 2-D system, which is called local reachability index $\left(I_{L R}\right)$ of that system, was recently tackled by means of the use of a suitable composition table. The greatest index $I_{L R}$ obtained in the previous literature was $n+3(\lfloor n / 2\rfloor)^{2}$ for some appropriated values of $n$. Taking as a basis both a combinatorial approach of such systems and the construction of suitable geometric sets in the plane, an upper bound on $I_{L R}$ depending on the dimension $n$ for a new family of systems is characterized. The 2-D influence digraph of this family of order $n \geq 6$ consists of two subdigraphs corresponding to a unique source $s$. The first one is a cycle involving the first $n_{1}$ vertices and is connected to the another subdigraph through the 1 -arc $\left(2, n_{1}+n_{2}\right)$, being the natural numbers $n_{1}$ and $n_{2}$ such that $n_{1}>n_{2} \geq 2$ and $n-n_{1}-n_{2} \geq 1$. The second one has two main cycles, a cycle where only the remaining vertices $n_{1}+1, \ldots, n$ appear and a cycle containing only the vertices $n_{1}+1, \ldots, n_{1}+n_{2}-1$. Moreover, the last vertices are connected through the 2 -arc $\left(n_{1}+n_{2}-1, n\right)$. Furthermore, if $n \geq 12$ and is a multiple of 3 , for appropriate $n_{1}$ and $n_{2}$, the $I_{L R}$ of that family is at least
\end{abstract}

"An earlier version of this paper was presented at the Conference Linear Algebra, Matrix Analysis and Applications. ALAMA2018, held in Sant Joan dAlacant on May/June 2018".

Esteban Bailo, Josep Gelonch

Department de Matemàtica,

Universitat de Lleida,

Av. de l'Alcalde Rovira Roure, 191. 25198 Lleida. Spain.

E-mail: ebailo@matematica.udl.es, jgelonch@matematica.udl.es

S. Romero-Vivó

Instituto de Matemática Multidisciplinar,

Universitat Politècnica de València,

Camino de Vera, 14. 46022 Valencia. Spain.

E-mail: sromero@imm.upv.es

and Centro de Investigación Biomédica en Red de Diabetes y Enfermedades Metabólicas Asociadas (CIBERDEM), Instituto de Salud Carlos III, 28029 Madrid, Spain 
cubic, exactly, it must be $\frac{n^{3}+9 n^{2}+45 n+108}{27}$, which shows that some local states can be deterministically reached much further than initially proposed in the literature.

Keywords Positive two dimensional (2-D) systems · Fornasini-Marchesini models · Hurwitz products · Influence digraph · Local reachability index . Composition table

Mathematics Subject Classification (2010) 05C99 - 15A48 - 93 B03 . $93 \mathrm{C} 55$

\section{Introduction}

In this paper, we focus our attention on positive two-dimensional (2-D) systems described by the Fornasini-Marchesini state-space model (see [1] and [26]) which is as follows:

$$
x_{i+1, j+1}=A_{1} x_{i+1, j}+A_{2} x_{i, j+1}+B_{1} u_{i+1, j}+B_{2} u_{i, j+1}
$$

with local states $x_{.,} \in \mathbb{R}_{+}^{n}$, inputs $u,, \in \mathbb{R}_{+}^{m}$, state matrices $A_{1}, A_{2} \in \mathbb{R}_{+}^{n \times n}$, input matrices $B_{1}, B_{2} \in \mathbb{R}_{+}^{n \times m}$ and initial global state $\chi_{0}:=\left\{x_{h, k}:(h, k) \in \mathcal{C}_{0}\right\}$ being $\mathcal{C}_{0}:=\{(h, k): h, k \in \mathbb{Z}, h+k=0\}$. Let us denote these systems by $\left(A_{1}, A_{2}, B_{1}, B_{2}\right)$.

It is well-known that the nonnegativity condition yields a different treatment of these control systems based upon the theory of nonnegative matrices. In fact, in the last decade has been an increasing interest in the theory of positive systems (for example, see [4], [7], [8], [14] and [19]), which appear in many different real situations such as in economics, biological, environmental and chemical processes, among others (see [25], [33], [37], [38] and [43]). A considerable amount of literature has been published on the analysis of structural properties and more specifically on the reachability property (see [12], [13], [17], [23], [24], [32], [42] and [44]). In fact, it is still the subject of a detailed investigation (see [3], [5], [6], [15], [18], [22], [34], [35], [36], [40], [45] and [46], among others).

When dealing with positive 2-D discrete state-space models, the structural property of reachability is introduced both in a local form, which refers to single local states, and in a global form (see [29], [31] and [41]). Throughout this work, we pay heed to the study of the smallest number of steps needed to reach all local states of a system, that is, the local reachability index of that systems. This index plays an important role because it enables us to determine in a finite number of steps whether a system is locally reachable or not as well as to know how many separations sets at most are needed to achieve the states. Previous studies such as [10], [11], [16] and references therein, [20] and [21] have studied and reported that the reachability index for positive 1-D systems is always bounded by the dimension $n$. However, several attempts in two dimensional case (see [1], [2] and [9] and references therein) have been made to obtain a general formula depending on $n$ on the local reachability 
index for any positive 2-D systems. So far, despite the use of graph-theoretic techniques, to solve this question seems to be a nontrivial goal to accomplish due to its computational complexity (see [30]). In [39], an alternative method for determination of a lower reachability index and reachability index space for a positive second Fornasini-Marchesini model is proposed.

To the best of the authors' knowledge, the most promising tool used until now to tackle the study of the local reachability index is the composition table introduced in [2], which enables us to calculate easily the Hurwitz products involved in a reachability matrix in $k$-steps for a positive integer $k$. Besides that, from that instrument, it is possible to construct suitable geometric sets in the plane covering the greatest number of entries possible on such the table. This new geometrical approach allows us to reduce the positions on the table where local states can be deterministically reached and therefore, to construct families of systems with greatest local reachability index, even with a cubic local reachable index, as shown in this paper. More specifically, from now on, the local reachability index for a special class of positive 2-D systems is completely characterized in terms of the order $n \geq 6$ as well as of the natural numbers $n_{1}$ and $n_{2}$ determining their corresponding influence digraphs. Additionally, it is deduced that the absolute maximum value of $I_{L R}$ for the associated system family $\mathcal{F}$ is obtained if $n \geq 12, n_{2}=\left\lfloor\frac{n+4}{3}\right\rfloor$ and $n_{1}=n_{2}+1$. Besides that, if $n$ is also a multiple of $3, I_{L R}$ is cubic, exactly $\frac{n^{3}+9 n^{2}+45 n+108}{27}$, that in comparison with the previously suggested greatest upper bound $n+3(\lfloor n / 2\rfloor)^{2}$ (see $[2])$ is considerably larger and it seems to be more much realistic, although almost surely it can be refined on the whole.

The paper has been structured as follows: Section II introduces some notations and basic definitions. In Section III, a new family of positive 2-D systems whose local reachability index largely exceeds the last upper bound given in the literature is provided. Finally, in Section IV, a bound on that local reachability index is studied, which even turn to be cubic under suitable conditions.

\section{Preliminaries}

For the convenience of the reader, we repeat the relevant material from [2], thus making our exposition self-contained.

We denote by $\lfloor z\rfloor$ the lower integer-part of $z \in \mathbb{R}$ and by $c_{j}(A)$ the $j$ th column of $A \in \mathbb{R}^{n \times n}$.

Definition 1 (see [27]) The Hurwitz products of the $n \times n$ matrices $A_{1}$ and $A_{2}$ are defined as follows:

- $A_{1}{ }^{i} \sqcup^{j} A_{2}=0, \quad$ when either $i$ or $j$ is negative,

- $A_{1}{ }^{i} \sqcup^{0} A_{2}=A_{1}^{i}, \quad$ if $i \geq 0, \quad A_{1}{ }^{0} \sqcup^{j} A_{2}=A_{2}^{j}, \quad$ if $j \geq 0$,

- $A_{1}{ }^{i} \sqcup^{j} A_{2}=A_{1}\left(A_{1}{ }^{i-1} \sqcup^{j} A_{2}\right)+A_{2}\left(A_{1}{ }^{i} \sqcup^{j-1} A_{2}\right)$, if $i, j>0$.

Observe that $\sum_{i+j=\ell} A_{1}{ }^{i} \sqcup^{j} A_{2}=\left(A_{1}+A_{2}\right)^{\ell}$. 
Definition 2 (see [29]) A 2-D state-space model (1) is (positively) locally reachable if, upon assuming $\chi_{0}=0$, for every state $x^{*} \in \mathbb{R}_{+}^{n}$, there exists $(h, k) \in \mathbb{Z} \times \mathbb{Z}, h+k>0$, and a nonnegative input sequence $u .$, such that $x_{h, k}=x^{*}$. When so, the state is said to be (positively) reachable in $h+k$ steps. The smallest number of steps allowing to reach all nonnegative local states represents the local reachability index $I_{L R}$ of such a system.

Characterizations of the local reachability of a positive 2-D system $\left(A_{1}, A_{2}\right.$, $\left.B_{1}, B_{2}\right)$ can be established in terms of its reachability matrix. The reachability matrix in $k$-steps is given by

$$
\begin{aligned}
& \mathcal{R}_{k}=\left[\begin{array}{llllllll}
B_{1} & B_{2} & A_{1} B_{1} & A_{1} B_{2}+A_{2} B_{1} & A_{2} B_{2} & A_{1}^{2} B_{1} & \cdots & A_{2}^{k-1} B_{2}
\end{array}\right] \\
& =\left[\left(A_{1}^{i-1} \sqcup^{j} A_{2}\right) B_{1}+\left(A_{1}{ }^{i} \sqcup \amalg^{j-1} A_{2}\right) B_{2}\right]_{i, j \geq 0,0<i+j \leq k}
\end{aligned}
$$

where $k \in \mathbb{N}$. It is known (see [29]) that the local reachability property holds if and only if there are $n$ pairs $\left(h_{i}, k_{i}\right) \in \mathbb{N} \times \mathbb{N}, i=1, \ldots, n$, and $n$ indices $j=$ $j(i) \in\{1,2, \ldots, m\}$ such that $\left(A_{1}^{h_{i}-1} \sqcup^{k_{i}} A_{2}\right) c_{j}\left(B_{1}\right)+\left(A_{1}{ }^{h_{i}} \cup^{k_{i}-1} A_{2}\right) c_{j}\left(B_{2}\right)$ is a positive $i$ th monomial vector, that is, there exists $k \in \mathbb{N}$ such that $\mathcal{R}_{k}$ contains an $n \times n$ monomial matrix. If so,

$$
\begin{aligned}
& I_{L R}=\max _{i} \min _{h_{i}, k_{i}}\left\{h_{i}+k_{i} \mid\left(A_{1}^{h_{i}-1} \sqcup^{k_{i}} A_{2}\right) c_{j}\left(B_{1}\right)+\left(A_{1}^{h_{i}} \sqcup^{k_{i}-1} A_{2}\right) c_{j}\left(B_{2}\right)\right. \\
& \text { is an } i-\text { monomial vector for some } j=j(i)\}
\end{aligned}
$$

We recall that a positive $i$ th monomial vector (or simply $i$-monomial vector throughout this paper) is a positive multiple of the $i$ th unit vector $e_{i}$ of $\mathbb{R}^{n}$. Similarly, a monomial matrix is a nonsingular matrix having a unique positive entry in each row and column.

From now on, we consider a family of colored digraphs constructed from the matrices of the system $\left(A_{1}, A_{2}, B_{1}, B_{2}\right)$ as follows:

Definition 3 (see [29]) Associated with system (1), a directed digraph called 2-D influence digraph is defined. It is denoted by $\mathcal{D}^{(2)}\left(A_{1}, A_{2}, B_{1}, B_{2}\right)$ and it is given by $\left(S, V, \mathcal{A}_{1}, \mathcal{A}_{2}, \mathcal{B}_{1}, \mathcal{B}_{2}\right)$, where $S=\left\{s_{1}, s_{2}, \ldots, s_{m}\right\}$ is the set of sources, $V=\left\{v_{1}, v_{2}, \ldots, v_{n}\right\}$ is the set of vertices, $\mathcal{A}_{1}$ and $\mathcal{A}_{2}$ are subsets of $V \times V$ whose elements are named $\mathcal{A}_{1}$-arcs and $\mathcal{A}_{2}$-arcs (or simply 1-arcs and 2-arcs), respectively, while $\mathcal{B}_{1}$ and $\mathcal{B}_{2}$ are subsets of $S \times V$ whose elements are tagged $\mathcal{B}_{1}$-arcs and $\mathcal{B}_{2}$-arcs (or simply 1 -arcs and 2-arcs), respectively. There is an $\mathcal{A}_{1}$-arc $\left(\mathcal{A}_{2}\right.$-arc $)$ from $v_{j}$ to $v_{i}$ if and only if the $(i, j)$ th entry of $A_{1}\left(A_{2}\right)$ is nonzero. There is a $\mathcal{B}_{1}$-arc $\left(\mathcal{B}_{2}\right.$-arc $)$ from $s_{\ell}$ to $v_{i}$ if and only if the $(i, \ell)$ th entry of $B_{1}\left(B_{2}\right)$ is nonzero.

Definition $4 \mathrm{~A}$ path in $\mathcal{D}^{(2)}\left(A_{1}, A_{2}, B_{1}, B_{2}\right)$ from $v_{i_{1}}$ to $v_{i_{p}}$ is an alternating sequence of vertices and $\operatorname{arcs}\left\{v_{i_{1}},\left(v_{i_{1}}, v_{i_{2}}\right), v_{i_{2}}, \ldots,\left(v_{i_{p-1}}, v_{i_{p}}\right), v_{i_{p}}\right\}$ such that $\left(v_{i_{1}}, v_{i_{2}}\right) \in \mathcal{A}_{1} \cup \mathcal{A}_{2} \cup \mathcal{B}_{1} \cup \mathcal{B}_{2}$ and $\left(v_{i_{k}}, v_{i_{k+1}}\right) \in \mathcal{A}_{1} \cup \mathcal{A}_{2}$ for all $k=2, \ldots, p-1$. A path is termed closed if the initial and final vertices coincide. In accordance with reference [29], an $s_{\ell}$-path is a path where $v_{i_{1}}=s_{\ell}$. The path length is defined to be equal to the number of arcs it contains, that is, $p+q$ being $p(q)$ the number of 1 -arcs $(2$-arcs) occurring in the path $\mathcal{P}$. Furthermore, the pair 
$(p, q)$ is called the composition of $\mathcal{P}$, a circuit is a closed path and finally, if each vertex appears exactly once as the first vertex of an arc, then the circuit is said to be a cycle.

Remark 1 Given $\mathcal{P} \equiv\left\{v_{i_{1}},\left(v_{i_{1}}, v_{i_{2}}\right), v_{i_{2}}, \ldots, v_{i_{p}}\right\}$ and $\mathcal{Q} \equiv\left\{v_{j_{1}},\left(v_{j_{1}}, v_{j_{2}}\right), \ldots\right.$, $\left.\left(v_{j_{q}-1}, v_{j_{q}}\right), v_{i_{q}}\right\}$ two paths such that $v_{i_{p}}=v_{j_{1}}$, we denote the path from $v_{i_{1}}$ to $v_{j_{q}}$ obtained by concatenating $\mathcal{P}$ and $\mathcal{Q}$ briefly by $\mathcal{P} \sqcup \mathcal{Q}$. Besides that, if $\mathcal{C}$ is a cycle, from now on, $\eta \mathcal{C}$ stands for the circuit resulting from doing $\eta$ laps around the cycle, $\eta$ being a positive integer.

Remark 2 In the sequel, we will write an 1-arc (2-arc) connecting two consecutive vertices $v_{k}$ and $v_{k+1}$ simply as $k \longrightarrow k+1(k--k+1)$ where $v_{1}, \ldots, v_{n}$ are relabeled as their subscripts.

Definition 5 (see [29]) If there exists an $s_{\ell}$-path in $\mathcal{D}^{(2)}\left(A_{1}, A_{2}, B_{1}, B_{2}\right)$ from the source $s_{\ell}$ to the vertex $v_{i}$, then $v_{i}$ is said to be reachable from $s_{\ell}$. Besides that, if for any $\ell \in\{1, \ldots, m\}$, all $s_{\ell}$-paths of composition $(p, q)$ end in the same vertex $v \in V$, then $v$ is said to be deterministically reachable from the source $s_{\ell}$ with composition $(p, q)$.

The shortest length of the $s_{\ell}$-paths deterministically reaching $v$ is called the $\ell$-index of $v$, i.e. $\mathcal{I}_{\ell}(v)=\min \left\{p+q \mid(p, q)\right.$ is the composition of an $s_{\ell}-$ path deterministically reaching $v\}$. Moreover, $I_{D}(v)=\min \left\{\mathcal{I}_{\ell}(v) \mid \ell=1, \ldots, m\right\}$ is named the determinant index of $v$. Hence, $I_{L R}=\max \left\{I_{D}(v) \mid v \in V\right\}$.

To facilitate the analysis of the local reachability index, a new tool was introduced in [2]. Exactly, from an algebraic point of view, a double-entry table generated just entering at each table entry $(i, j), i, j \in \mathbb{Z}_{+}$, the corresponding Hurwitz product $\left(A_{1}^{j-1} \sqcup^{i} A_{2}\right) B_{1}+\left(A_{1}^{j} \sqcup^{i-1} A_{2}\right) B_{2}$ involved in the reachability matrix in $k$-steps for a large enough $k$. This composition table is illustrated in Fig. 1.

Obviously, the aforementioned table can be interpreted from a combinatorial point of view. In fact, for every $\ell \in\{1, \ldots, m\}$, the list of vertices obtained by a $s_{\ell}$-path for a composition $(i, j)$ coincide with the subindices corresponding to the nonzero components of the $\ell$ th column of $\left(A_{1}^{i-1} \sqcup^{j} A_{2}\right) B_{1}+$ $\left(A_{1}{ }^{i} \cup^{j-1} A_{2}\right) B_{2}$, which is placed in the table entry $(j, i)$. Hence, for each source $s_{\ell}$, the vertices located in cell $(0,1)((1,0))$ are associated with the nonzero components of the corresponding column of the matrix $B_{1}\left(B_{2}\right)$. For the remaining table entries, a vertex $v$ can appear in the position $(j, i)$ on the table only if there exists at least either an 1-arc directed toward $v$ from a vertex in cell $(j, i-1)$ or a 2 -arc directed toward $v$ from a vertex in position $(j-1, i)$.

Remark 3 Note that a vertex is deterministically reached if and only if it can be located on an table entry without no other vertices.

Applying the theory of either positive 1-D systems or nonnegative matrices, let us deduce the following simple lemmas, which link the analysis of the reachability property of positive $1-\mathrm{D}$ systems to that of positive 2-D systems: 


\begin{tabular}{|c|c|c|c|c|c|}
\hline$q^{p}$ & 0 & 1 & 2 & 3 & $\ldots$ \\
\hline 0 & & $B_{1}$ & $A_{1} B_{1}$ & $A_{1}^{2} B_{1}$ & $\ldots$ \\
\hline 1 & $B_{2}$ & $A_{2} B_{1}+A_{1} B_{2}$ & $\begin{array}{c}A_{2} A_{1} B_{1} \\
+ \\
A_{1}\left(A_{1} B_{2}+A_{2} B_{1}\right)\end{array}$ & $\begin{array}{c}\left(A_{1}^{2} \varpi^{1} A_{2}\right) B_{1} \\
+ \\
\left(A_{1}^{3} \varpi^{0} A_{2}\right) B_{2}\end{array}$ & $\cdots$ \\
\hline 2 & $A_{2} B_{2}$ & $\begin{array}{c}A_{1} A_{2} B_{2} \\
+ \\
A_{2}\left(A_{2} B_{1}+A_{1} B_{2}\right)\end{array}$ & $\begin{array}{c}\left(A_{1}^{1} \varpi^{2} A_{2}\right) B_{1} \\
+ \\
\left(A_{1}^{2} \varpi^{1} A_{2}\right) B_{2}\end{array}$ & $\begin{array}{c}\left(A_{1}^{2} \amalg^{2} A_{2}\right) B_{1} \\
+ \\
\left(A_{1}^{3} \varpi^{1} A_{2}\right) B_{2}\end{array}$ & $\ldots$ \\
\hline 3 & $A_{2}^{2} B_{2}$ & $\begin{array}{c}\left(A_{1}^{0} \uplus^{3} A_{2}\right) B_{1} \\
+ \\
\left(A_{1}^{1} \varpi^{2} A_{2}\right) B_{2}\end{array}$ & $\begin{array}{c}\left(A_{1}^{1} \uplus^{3} A_{2}\right) B_{1} \\
+ \\
\left(A_{1}^{2} \varpi^{2} A_{2}\right) B_{2}\end{array}$ & $\begin{array}{c}\left(A_{1}^{2} \amalg^{3} A_{2}\right) B_{1} \\
+ \\
\left(A_{1}^{3} \sqcup^{2} A_{2}\right) B_{2}\end{array}$ & $\ldots$ \\
\hline$\vdots$ & $\vdots$ & $\vdots$ & $\vdots$ & $\vdots$ & $\ddots$ \\
\hline
\end{tabular}

Fig. 1 Composition Table.

Lemma 1 (see Theorem 1 in [21]) If an $\ell$-monomial vector appears in the position $(0, i)((j, 0))$ on the table where $i>n(j>n)$, then such an $\ell$ monomial vector must necessarily be found on the preceding positions $(0, \ell)$ $((\ell, 0))$ for some $\ell \in\{1, \ldots, n\}$.

Lemma 2 (see Lemma 2 in [44]) If an $\ell$-monomial vector appears in the position $(j, i)$ on the table where $j \cdot i \geq 1$, then such an $\ell$-monomial vector must be necessarily an $\ell$-monomial column of the matrix $\left[A_{1} \mid A_{2}\right]$. Besides that, if an $\ell$-monomial vector comes up in the position $(0, i)((j, 0))$ on the table where $i>1(j>1)$, then such an $\ell$-monomial vector must be necessarily an $\ell$-monomial column of the matrix $A_{1}\left(A_{2}\right)$.

Given any positive 2 -D system $\left(A_{1}, A_{2}, B_{1}, B_{2}\right)$, let us define $\left(A_{1}, A_{2}, B_{1}, O\right)$ (similarly, $\left(A_{1}, A_{2}, O, B_{2}\right)$ ) as the $2-\mathrm{D}$ system derived from the initial system maintaining the same state and control matrices except for $B_{2}\left(B_{1}\right)$ that is replaced by the zero matrix $O$ of an appropriate size. Clearly, taking into account the definition of the Hurwitz Products, the following elementary lemma can be formulated:

Lemma 3 The composition table pertaining to the system $\left(A_{1}, A_{2}, B_{1}, B_{2}\right)$ is the overlapping, entry by entry, of the composition tables associated with the systems $\left(A_{1}, A_{2}, B_{1}, O\right)$ and $\left(A_{1}, A_{2}, O, B_{2}\right)$.

In order to get families with a greater local reachability index, let us establish connections between $I_{L R}$ of a system $\left(A_{1}, A_{2}, B_{1}, B_{2}\right)$ and the related indices of the associated systems $\left(A_{1}, A_{2}, B_{1}, O\right)$ and $\left(A_{1}, A_{2}, O, B_{2}\right)$.

Lemma 4 Suppose that $\left(A_{1}, A_{2}, B_{1}, B_{2}\right),\left(A_{1}, A_{2}, B_{1}, O\right)$ and $\left(A_{1}, A_{2}, O, B_{2}\right)$ are locally reachable positive 2-D systems then, $I_{L R}$ of $\left(A_{1}, A_{2}, B_{1}, B_{2}\right)$ is greater than or equal to the corresponding indices of both remaining systems. 

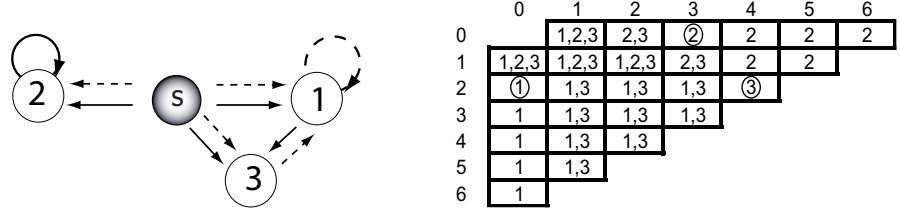

Fig. 2 Digraph and composition table in connection with example 1.
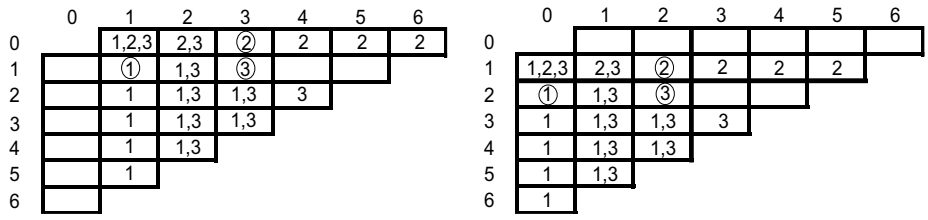

Fig. 3 Composition table as regard to $\left(A_{1}, A_{2}, B_{1}, O\right)$ and $\left(A_{1}, A_{2}, O, B_{2}\right)$, respectively.

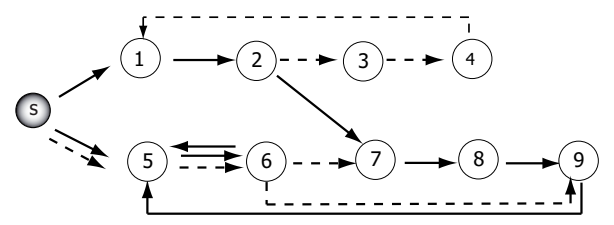

Fig. 4 Digraph as regards to the system examined in example 2.

However, it is worth pointing out an instance where strict inequality holds.

Example 1 Let us take the matrices $A_{1}=\left[e_{3}\left|e_{2}\right| 0\right] \in \mathbb{R}_{+}^{3 \times 3}, A_{2}=\left[e_{1}|0| e_{1}\right] \in$ $\mathbb{R}_{+}^{3 \times 3}$ and $B_{1}=B_{2}=\left[e_{1}+e_{2}+e_{3}\right] \in \mathbb{R}_{+}^{3 \times 1}$ which define a system (1) whose 2-D influence digraph and composition table are represented in Fig. 2.

Now, we construct the composition tables of $\left(A_{1}, A_{2}, B_{1}, O\right)$ and $\left(A_{1}, A_{2}, O, B_{2}\right)$ from the initial one, which are given in Fig. 3. We remark that both systems are locally reachable and their associated local reachability indices are equal to 4 while the original system is also locally reachable but $I_{L R}=6$, which is strictly greater than the previous ones.

In order to illustrate the way of analyzing the local reachability property for positive 2-D systems through their composition table, let us present the following example.

Example 2 The positive 2-D system $\left(A_{1}, A_{2}, B_{1}, B_{2}\right)$ of order $n=9$ described by the quadruple

$\left(\left[e_{2}\left|e_{7}\right| 0|0| e_{6}\left|e_{5}\right| e_{8}\left|e_{9}\right| e_{5}\right],\left[0\left|e_{3}\right| e_{4}\left|e_{1}\right| e_{6}\left|e_{7}+e_{9}\right| 0|0| 0\right],\left[e_{1}+e_{5}\right],\left[e_{5}\right]\right)$

has a 2-D influence digraph as given in Fig. 4 as well as a composition table as partially displayed in Fig. 5 . 


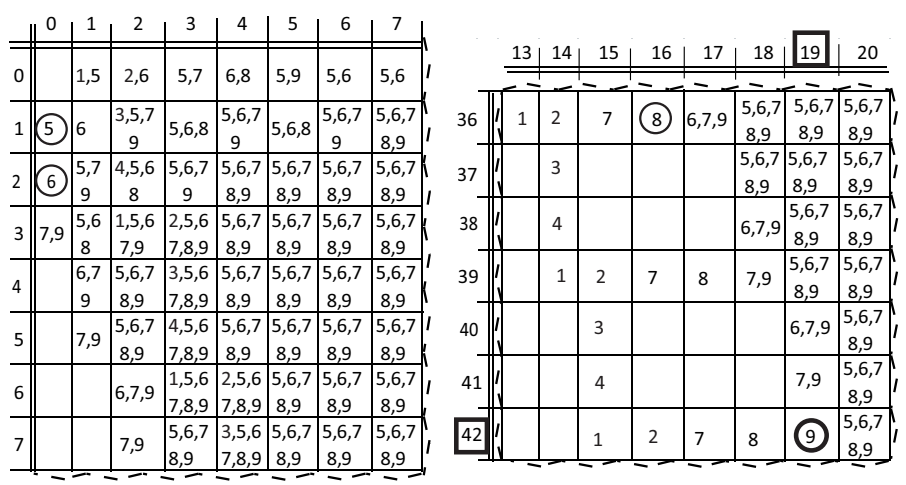

Fig. 5 Composition table associated with example 2.

In short, let us represent the cycles $\mathcal{C}_{1} \equiv\{1 \rightarrow 2 \rightarrow 3 \rightarrow 4 \rightarrow-\rightarrow 1\}$, $\mathcal{C}_{2} \equiv\{5 \rightarrow 6 \rightarrow 9 \longrightarrow 5\}, \mathcal{C}_{3} \equiv\{5 \longrightarrow 6 \longrightarrow 5\}$ and the path $\mathcal{P}_{1} \equiv\{7 \longrightarrow$ $8 \longrightarrow 9\}$.

A quick glance at Fig. 5 reveals the determinant index for each one of the vertices along with the composition for which the corresponding $s$-path deterministically reaches it, that is,

$$
\begin{aligned}
& I_{D}\left(v_{5}\right)=1, \quad I_{D}\left(v_{6}\right)=2, I_{D}\left(v_{1}\right)=25 \\
& I_{D}\left(v_{4}\right)=28, I_{D}\left(v_{3}\right)=31, I_{D}\left(v_{2}\right)=34 \\
& I_{D}\left(v_{7}\right)=43, I_{D}\left(v_{8}\right)=52, I_{D}\left(v_{9}\right)=61 .
\end{aligned}
$$

Besides that, the system $\left(A_{1}, A_{2}, B_{1}, B_{2}\right)$ is locally reachable and $I_{L R}=61$.

However, to entirely construct the composition table is not useful from a computational point of view. Thus, let us significantly reduce the number of vertices appearing in the entries on the table. Focusing on the structure of the digraph given in Fig. 4, we emphasize that if there is some table position $(q, p)$ involving at least one vertex in $\mathcal{C}_{3}$ then all cells $\left(q, p_{2}\right)$ with $p_{2} \geq p$ are occupied by at least vertex 5 or 6 . Hence, we can assure that if there is some position on the composition table involving at least one vertex in $\mathcal{C}_{3}$, all entries to its right-hand side in that same row also contains at least vertex 5 or vertex 6 . In addition, no new vertices can be deterministically reached in all these entries. Thus, let us omit vertices 5 and 6 in the corresponding positions on the table and let us blue paint such entries to mark this restricted zone associated with vertices in $\mathcal{C}_{3}$. Therefore, clearly, no vertices forming the cycle $\mathcal{C}_{1}$ can be deterministically reached while appear in entries inside the blue zone. As a consequence, let us use a schematic representation of the composition table (see Fig. 6) where only some vertices belonging to $\mathcal{C}_{1}, \mathcal{C}_{2}$ and $\mathcal{P}_{1}$ are indicated. Obviously, this new visual model is simpler than the previous one.

Furthermore, this new approach allows us to limit the set of entries on the table where a vertex can be deterministically reached or alternatively, to 


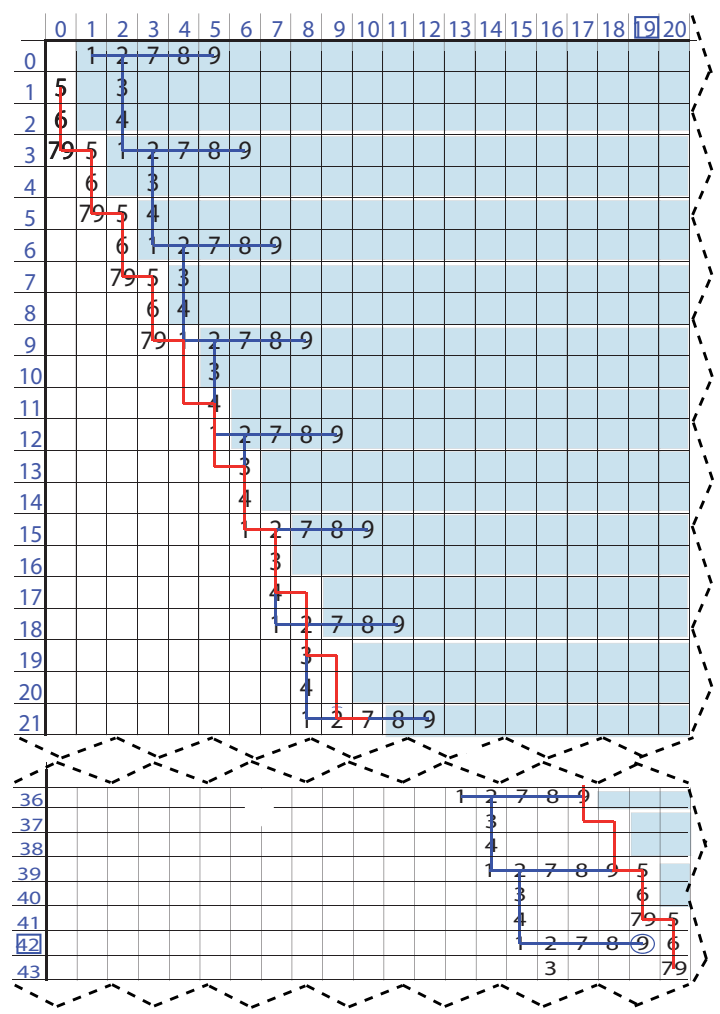

Fig. 6 A sketch for the composition table of example 2.

define broad regions with all their cells completely filled with vertices and in this way, to study how far, as a maximum, we have to go to deduce $I_{L R}$.

\section{Local reachability index for a special class of systems}

From the sketch given in example 2, let us construct a new family of positive 2D systems whose local reachability index largely exceeds the last upper bound given in the literature (see $[2])$, that is, $n+3(\lfloor n / 2\rfloor)^{2}$ for some odd natural number $n \geq 5$. Namely, we consider a family $\mathcal{F}$ of $n$ th-order systems with $n \geq 6$ and whose influence digraph associated is depicted in Fig. 7 , being the natural numbers $n_{1}$ and $n_{2}$ such that $n_{1}>n_{2} \geq 2$ and $n-n_{1}-n_{2} \geq 1$.

Theorem 1 Every $n$ th-order system of family $\mathcal{F}$ with $n \geq 6$, the natural numbers $n_{1}$ and $n_{2}$ such that $n_{1}>n_{2} \geq 2$ and $n-n_{1}-n_{2} \geq 1$ and whose influence digraph associated is depicted in Fig. 7 is (positively) locally reachable, and furthermore,

$$
I_{L R} \leq \frac{(3+k) n_{2}\left(n_{1}-1\right)+n_{1}^{2}}{n_{1}-n_{2}}, \text { with } k=n-n_{1}-n_{2} .
$$




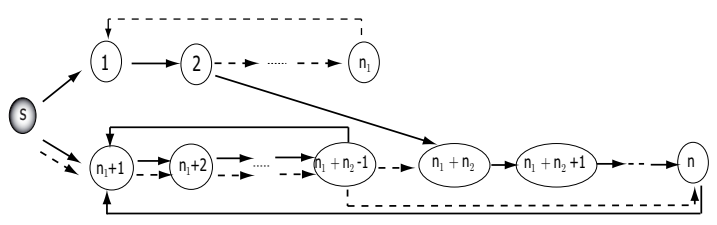

Fig. 7 Digraph for family $\mathcal{F}$.

Proof To reduce notation, we denote the path $\left\{n_{1}+n_{2} \longrightarrow n_{1}+n_{2}+1 \longrightarrow\right.$ $\cdots \longrightarrow n\}$ of composition $(k, 0)$ (see Fig. 7 ) by $\mathcal{P}_{1}$ and also, we define the cycles $\mathcal{C}_{1} \equiv\left\{1 \longrightarrow 2 \rightarrow \cdots-\rightarrow n_{1} \rightarrow 1\right\}, \mathcal{C}_{2} \equiv\left\{n_{1}+1 \rightarrow \cdots-\rightarrow n_{1}+n_{2}-1 \rightarrow\right.$ $\left.n \longrightarrow n_{1}+1\right\}$ and $\mathcal{C}_{3} \equiv\left\{n_{1}+1 \longrightarrow \cdots \longrightarrow n_{1}+n_{2}-1 \longrightarrow n_{1}+1\right\}$ whose compositions are $\left(1, n_{1}-1\right),\left(1, n_{2}-1\right),\left(n_{2}-1,0\right)$, respectively.

By its composition table (see Fig. 8), we can notice the following facts:

- All the possible $s$-paths of composition $(0, j)$, with $1 \leq j \leq n_{2}-1$, terminate at vertex $n_{1}+j$, and therefore, we can affirm that $n_{1}+j$ is deterministically reachable with composition $(0, j)$. Thus, the vertices $n_{1}+1, n_{1}+2, \ldots, n_{1}+$ $n_{2}-1$ are deterministically reachable and their determinant index is smaller than or equal to $n_{2}-1$. Note that $\mathcal{C}_{3}$ is made up of those vertices, which have been represented as a white ellipse inside a shady rectangle on the composition table (see Fig. 8).

- Taking into account that $\mathcal{C}_{3}$ consist only of 1 -arcs, we can assure that if there is some position on the composition table involving at least one vertex in $\mathcal{C}_{3}$ then, the remaining cells to its right-hand side in that same row are also occupied by vertices in $\mathcal{C}_{3}$ (although omitted on the table, see step 2, Fig. 8). Moreover, as $\mathcal{C}_{3}$ is accessible from the source $s$ by using an 1 arc, it is clear that, without utilizing 2-arcs, there are no deterministically reachable vertices out of $\mathcal{C}_{3}$.

- At first, the vertices in $\mathcal{C}_{1}$ (illustrated in Fig. 8 by white stars except for vertex 2 , which is black, inside a lined rectangle) are reachable by $s$-paths needing more 1 -arcs than those in cycle $\mathcal{C}_{2}$, that is, they are placed on the composition table further to the right than those in $\mathcal{C}_{2}$. However, they are not deterministically reached since there are more vertices in the cells containing such vertices. Observe that if a vertex in $\mathcal{C}_{1}$ is placed on positions $(j, i)$ then it appears again in $\left(j+n_{1}-1, i+1\right)$, in $\left(j+2\left(n_{1}-1\right), i+2\right)$, and so on (depending on the number of laps done around the cycle $\left.\mathcal{C}_{1}\right)$. Moreover, all these positions on the table can be connected through a straight line (for instance, see Fig. 8, line $r_{3}$ associated with vertex 2). Similar reasonings can be applied to derive straight lines for vertices in $\mathcal{C}_{2}$ (see Fig. 8, line $r_{2}$ corresponding to vertex $n_{1}+n_{2}-1$ ), and for those in $\mathcal{P}_{1}$ (combining this path with the cycle $\mathcal{C}_{1}$, (see Fig. 8 , line $r_{1}$ corresponding to vertex $n$ )).

Obviously (see step 3, Fig. 8), taking into account that the number of 2arcs involved to cover the laps done around $\mathcal{C}_{2}\left(n_{1}>n_{2}\right)$ is greater than around $\mathcal{C}_{1}$, the lines $r_{2}$ and $r_{3}$ intersect, then, for a large enough number of laps, the vertices in $\mathcal{C}_{1}$ go out of the region of entries cover by vertices 


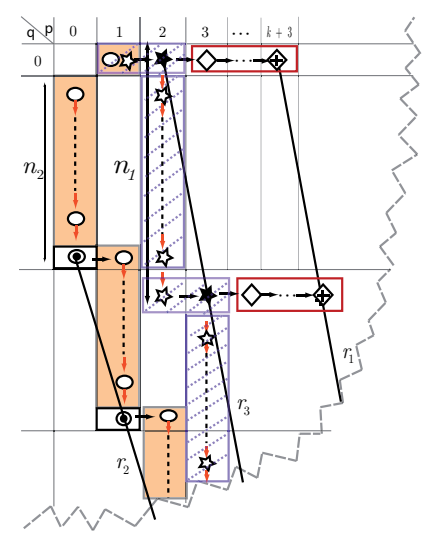

Step 1

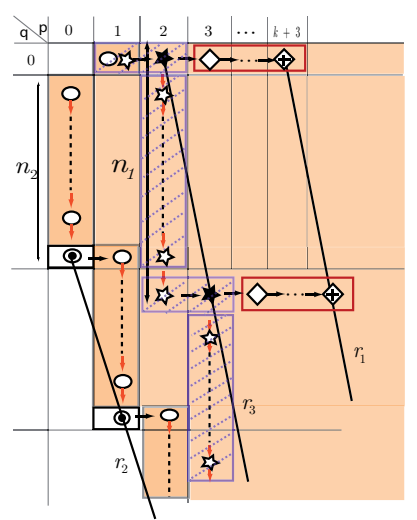

Step 2

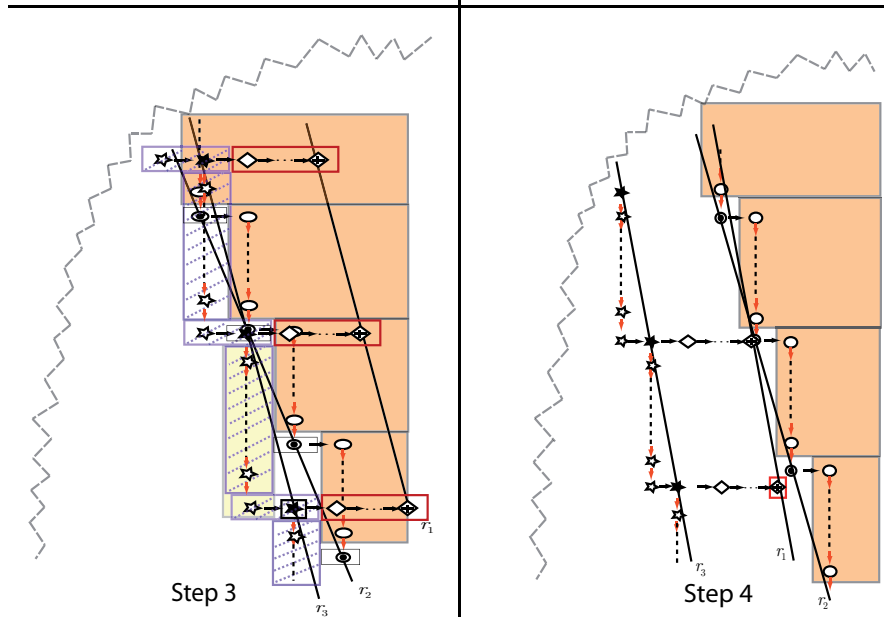

Fig. 8 Step 1:A sketch for the composition table of any system belonging to $\mathcal{F}$. Step $2: \mathcal{C}_{3}$ fills the cell rows with vertices in $\mathcal{C}_{3}$. Step 3: Precise moment in which the last vertex in $\mathcal{C}_{1}$ is deterministically reached. Step 4: Instant in which the last vertex in $\mathcal{P}_{1}$ is deterministically reached.

in $\mathcal{C}_{3}$. Thus, we can deterministically reach all vertices in $\mathcal{C}_{1}$. Furthermore, clearly, the last vertex deterministically reached in $\mathcal{C}_{1}$ is 2 .

- Clearly, using line $r_{1}$ instead of $r_{3}$ and reasoning as before, the vertices in $\mathcal{P}_{1}$ (shown by white diamonds) can be deterministically reached (in fact, after doing it the vertices in $\mathcal{C}_{1}$ ).

- Finally, analyzing the slope of each lines for vertices in $\mathcal{C}_{1}$ and $\mathcal{P}_{1}$, the last vertex deterministically reached is $n$ (see step 4, Fig. 8) and this event occurs after doing one more lap around cycle $\mathcal{C}_{1}$ subsequently to the intersection of lines $r_{1}$ and $r_{2}$.

Therefore, to determine the local reachability index for any system of this family, we can analyze the determinant index of vertex $n$. Namely, by seeing 
how the cycles $\mathcal{C}_{1}$ and $\mathcal{C}_{2}$ appear on the composition table, we have to reckon up the intersection point of the line $r_{1}$ that has slope $\left(n_{1}-1\right)$ and passes through the point $(3+k, 0)$ and the line $r_{2}$ that has slope $\left(n_{2}-1\right)$ and passes through the point $\left(0, n_{2}\right)$. Such a intersection point $(x, y)$ is the solution of the system

$$
\begin{aligned}
& y=\left(n_{1}-1\right)(x-(3+k)), \\
& y=n_{2}+\left(n_{2}-1\right) x,
\end{aligned}
$$

that is,

$$
\begin{aligned}
& x=\frac{(3+k)\left(n_{1}-1\right)+n_{2}}{n_{1}-n_{2}}, \\
& y=n_{2}+\left(n_{2}-1\right) \frac{(3+k)\left(n_{1}-1\right)+n_{2}}{n_{1}-n_{2}} .
\end{aligned}
$$

Since the only valid solutions are those that provide a composition, we must impose that $x, y \in \mathbb{N}_{0}$. Thus, doing one more lap around the cycle $\mathcal{C}_{1}$, we choose

$$
\begin{aligned}
& x_{0}=\left\lfloor\frac{(3+k)\left(n_{1}-1\right)+n_{1}}{n_{1}-n_{2}}\right\rfloor, \\
& y_{0}=\left(n_{1}-1\right)\left(x_{0}-(k+3)\right),
\end{aligned}
$$

and we can write $I_{D}(n)=x_{0}+y_{0}$, that is,

$$
I_{D}(n)=\left\lfloor\frac{(3+k)\left(n_{1}-1\right)+n_{1}}{n_{1}-n_{2}}\right\rfloor+\left(n_{1}-1\right)\left\lfloor\frac{(3+k)\left(n_{2}-1\right)+n_{1}}{n_{1}-n_{2}}\right\rfloor .
$$

If $\lambda_{1}$ and $\lambda_{2}$ stand for the lower integer-parts appearing in equation (3) then

$$
\begin{aligned}
& (3+k)\left(n_{1}-1\right)+n_{1}=\lambda_{1}\left(n_{1}-n_{2}\right)+t_{1} \\
& (3+k)\left(n_{2}-1\right)+n_{1}=\lambda_{2}\left(n_{1}-n_{2}\right)+t_{2}
\end{aligned}
$$

with $t_{1}$ and $t_{2} \in\left[0, n_{1}-n_{2}\right) \cap \mathbb{Z}_{+}$.

Subtracting these two equations, $(3+k)\left(n_{1}-n_{2}\right)=\left(\lambda_{1}-\lambda_{2}\right)\left(n_{1}-n_{2}\right)+$ $\left(t_{1}-t_{2}\right)$. Hence, $t_{1}=t_{2}$ and $(3+k)=\left(\lambda_{1}-\lambda_{2}\right)$. Plugging these equalities into (3), $I_{D}(n)=\lambda_{1}+\left(n_{1}-1\right) \lambda_{2}$, then

$$
I_{D}(n)=(3+k)+n_{1}\left\lfloor\frac{(3+k)\left(n_{2}-1\right)+n_{1}}{n_{1}-n_{2}}\right\rfloor .
$$

Consequently, using that $\lfloor x\rfloor \leq x$ and simplifying, we deduce the desired formula

$$
I_{D}(n) \leq \frac{(3+k) n_{2}\left(n_{1}-1\right)+n_{1}^{2}}{n_{1}-n_{2}} .
$$

Alternatively, for $n=n_{1}+n_{2}+k,(2)$ is rewritten as

$$
I_{L R} \leq \frac{\left(3+n-n_{1}-n_{2}\right) n_{2}\left(n_{1}-1\right)+n_{1}^{2}}{n_{1}-n_{2}} .
$$




\section{Maximum value of $I_{L R}$ for $\mathcal{F}$}

The bound given in (4) depends on $n_{1}$ and $n_{2}$ with the aforementioned restrictions. Now, for every $n \geq 6$ let us find the values of these variables that maximize such a index. With this aim, we define the following function

$$
f\left(n_{1}, n_{2}\right):=\frac{\left(3+n-n_{1}-n_{2}\right) n_{2}\left(n_{1}-1\right)+n_{1}^{2}}{n_{1}-n_{2}} .
$$

To analyze its behaviour, we make a change of variables $u=n_{1}+n_{2}, v=$ $n_{1}-n_{2}$. Let us denote $g(u, v):=f\left(\frac{u+v}{2}, \frac{u-v}{2}\right)$ and from (5), it follows that

$$
g(u, v)=\frac{1}{4 v}\left[(3+n-u)(u-v)(u+v-2)+(u+v)^{2}\right] .
$$

Thus, the partial derivative of $g(u, v)$ with respect to $u$ is

$$
\frac{\partial g(u, v)}{\partial u}=\frac{-1}{4 v}\left[3 u^{2}-2(6+n) u+2(3+n)-v^{2}\right] .
$$

Then, we can state that

$$
\frac{\partial g(u, v)}{\partial u}=0 \Leftrightarrow u=\frac{(6+n) \pm \sqrt{18+6 n+n^{2}+3 v^{2}}}{3} .
$$

As $u=\frac{(6+n)-\sqrt{18+6 n+n^{2}+3 v^{2}}}{3}$ does not satisfy that $u=n_{1}+n_{2} \geq 5$ for any suitable number $n$ (under the constraints), the value desired is $u_{0}(v)=$ $\frac{(6+n)+\sqrt{18+6 n+n^{2}+3 v^{2}}}{3}$.

Let us define $G(v):=g\left(u_{0}(v), v\right)=\frac{54+27 n+9 n^{2}+n^{3}+81 v+27 n v-9 n v^{2}+\left(n^{2}+6 n+18+3 v^{2}\right)^{\frac{3}{2}}}{54 v}$, then the derivative of $\mathrm{G}$ is,

$$
G^{\prime}(v)=-\frac{n^{3}+9 n^{2}+27 n+9 n v^{2}+54+\left(n^{2}+6 n+18-6 v^{2}\right) \sqrt{n^{2}+6 n+18+3 v^{2}}}{54 v^{2}} .
$$

Taking $Z(v)$ the numerator of $G^{\prime}(v)$ with changed sign, let us check that $Z(v)>0$ for every $v \in[0, n]$, equivalently, $G(v)$ is decreasing at $v$ if $v \in(0, n)$.

Initially, we calculate $Z^{\prime}(v)=-\frac{9 v\left[6\left(3+v^{2}\right)+n\left(6+n-2 \sqrt{n^{2}+6 n+18+3 v^{2}}\right)\right]}{\sqrt{n^{2}+6 n+18+3 v^{2}}}$. Notice

that $Z^{\prime}(v)=0$ has as real solutions, $v= \pm \sqrt{-3-n+\frac{n \sqrt{12+4 n+n^{2}}}{2 \sqrt{3}}}$ and $v=0$, and also $Z^{\prime \prime}(0)=9\left(2 n-\sqrt{n^{2}+6 n+18}\right)>0, \forall n \geq 4$, then, $Z(v)$ has a local minimum point at $v=0$.

On the one hand $Z(0)=54+27 n+9 n^{2}+n^{3}+\left(18+6 n+n^{2}\right)^{(3 / 2)}>0$, on the other hand

$$
Z(n)=10 n^{3}+9 n^{2}+27 n+54+\left(6 n+18-5 n^{2}\right) \sqrt{6 n+18+4 n^{2}}
$$

and for every $n \geq 3,\left(6 n+18-5 n^{2}\right)<0$, thus, we can affirm that $(6 n+18-$ $\left.5 n^{2}\right) \sqrt{6 n+18+4 n^{2}}$ is greater than $\left(6 n+18-5 n^{2}\right)(2 n+4)$. Hence, $Z(n)$ is 


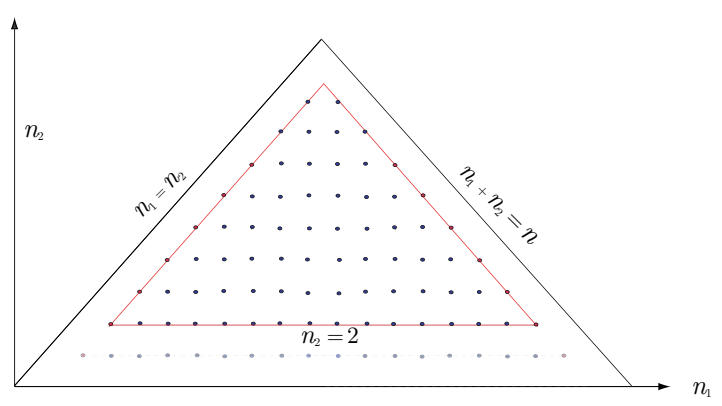

Fig. 9 Feasible region.

bounded from below by $n^{2}+87 n+126>0$ since $Z(n)>10 n^{3}+9 n^{2}+27 n+$ $54+\left(6 n+18-5 n^{2}\right)(2 n+4)=n^{2}+87 n+126>0$.

In short, $Z(v)>0$ for every $n \geq 4$ and $v \in[0, n]$, so we deduce that $G^{\prime}(v)<0$ and $G(v)$ is decreasing at $(0, n)$. Consequently, the maximum value of $G(v)$ is obtained for the minimum possible value of $v$, i.e., $v=1$. Thus, $n_{1}-n_{2}=1, n_{1}=n_{2}+1, k=n-1-2 n_{2}$ and plugging this equalities into (5), $f\left(n_{2}+1, n_{2}\right)=\left(3+n-2 n_{2}\right) n_{2}^{2}+2 n_{2}+1$.

Now, its derivative with respect to $n_{2}$ is $\frac{\partial f\left(n_{2}+1, n_{2}\right)}{\partial n_{2}}=2+6 n_{2}+2 n n_{2}-6 n_{2}^{2}$.

As a consequence, $\frac{\partial f\left(n_{2}+1, n_{2}\right)}{\partial n_{2}}=0$ iff $n_{2}=\frac{3+n \pm \sqrt{n^{2}+6 n+21}}{6}$.

By the evaluation of the second derivate, $f\left(n_{2}+1, n_{2}\right)$ has a relative maximum point at $n_{2}^{\prime}=\frac{1}{6}\left[3+n+\sqrt{n^{2}+6 n+21}\right]$ and a local minimum point ${ }^{1}$ at $n_{2}^{\prime \prime}=\frac{1}{6}\left[3+n-\sqrt{n^{2}+6 n+21}\right]$. In search of absolute maximum points, we must know when the local maximum point is a feasible solution since our solutions must belong to the region $\mathcal{D}=\left\{\left(n_{1}, n_{2}\right) \in \mathbb{N}^{2} \mid n_{1}+n_{2}<n, n_{1}>n_{2} \geq 2\right\}$ whose illustration is given in Fig. 9.

To determine whether the relative maximum point is in the aforementioned region, we bound from below and from above $n_{2}^{\prime}$, if possible, by using values in $\mathcal{D}$. Observe that $n^{2}+6 n+21$ can be expressed either as $(n+3)^{2}+12$ or as $(n+4)^{2}-2 n+5$ then,

$$
\frac{n}{3}+1<n_{2}^{\prime}=\frac{3+n+\sqrt{n^{2}+6 n+21}}{6}<\frac{n}{3}+\frac{7}{6}
$$

Let us analyze this inequality depending on the possible values of $n$. Namely, for the sake of brevity, we study only if $n=3 p-1$ with $p \in \mathbb{N}$ since similar reasoning as in this case can be applied to the cases $n=3 p$ and $n=3 p+1$.

If $n=3 p-1$, the inequalities given in (6) are reduced to $p+\frac{2}{3}<n_{2}^{\prime}<p+\frac{5}{6}$. Hence, either $n_{2}=p$ or $n_{2}=p+1$ provides the maximum point. Thus, it is

1 Note that $n_{2}^{\prime \prime}<1$ for every $n$ and so, it would always be out of $\mathcal{D}$. 
sufficient to evaluate $f$ for such values,

$$
\begin{aligned}
f(p+1, p) & =(p+2) p^{2}+2 p+1, \\
f(p+2, p+1) & =p(p+1)^{2}+2(p+1)+1 .
\end{aligned}
$$

As $f(p+2, p+1)-f(p+1, p)=p+2>0$, we choose $n_{2}=p+1$ (in the remaining cases too).

Summarising, for every $n \in[3 p-1,3 p+1]$ the natural number leading to the maximum value of $f\left(n_{2}+1, n_{2}\right)$ is $n_{2}=p+1$ and it can be written as $n_{2}=\left\lfloor\frac{n+1}{3}\right\rfloor+1=\left\lfloor\frac{n+4}{3}\right\rfloor$.

Clearly, this number $n_{2}$ ( $f$ takes its maximum value only if $\left.n_{1}=n_{2}+1\right)$ must meet the condition $\left(n_{2}+1, n_{2}\right) \in \mathcal{D}$. Hence, we must impose that $2 n_{2}+1 \leq$ $n-1$ and therefore,

$$
2\left\lfloor\frac{n+4}{3}\right\rfloor \leq n-2 \Leftrightarrow\left\lfloor\frac{n+4}{3}\right\rfloor \leq \frac{n}{2}-1,
$$

which holds for every $n \geq 12$. Besides that if $6 \leq n \leq 11$, the maximum values of $f\left(n_{1}, n_{2}\right)$ for $n_{1}=n_{2}+1$ are attained at the boundary of $\mathcal{D}$ and so, $n_{2}=\left\lfloor\frac{n}{2}\right\rfloor-1$.

As a result, the bound obtained for the local reachability index of systems in $\mathcal{F}$ is given by

$$
I_{L R} \leq \begin{cases}f\left(\left\lfloor\frac{n}{2}\right\rfloor,\left\lfloor\frac{n}{2}\right\rfloor-1\right) & \text { if } \quad 6 \leq n \leq 11 \\ f\left(\left\lfloor\frac{n+7}{3}\right\rfloor,\left\lfloor\frac{n+4}{3}\right\rfloor\right) & \text { if } \quad n \geq 12\end{cases}
$$

that is,

$$
I_{L R} \leq \begin{cases}\left(4+n-2\left\lfloor\frac{n}{2}\right\rfloor\right)\left(\left\lfloor\frac{n}{2}\right\rfloor-1\right)^{2}+\left\lfloor\frac{n}{2}\right\rfloor^{2} & \text { if } \quad 6 \leq n \leq 11 \\ (n+3)\left\lfloor\frac{n+4}{3}\right\rfloor^{2}-2\left\lfloor\frac{n+4}{3}\right\rfloor^{3}+2\left\lfloor\frac{n+4}{3}\right\rfloor+1 & \text { if } \quad n \geq 12 .\end{cases}
$$

Proposition 1 For every system of $\mathcal{F}$ with $n \geq 12$, its local reachability index is bounded by

$$
I_{L R} \leq \frac{1}{27}\left(n^{3}+9 n^{2}+45 n+108\right) .
$$

Furthermore, the equality is achieved for those systems satisfying that $n$ is a multiple of $3, n_{1}=\left\lfloor\frac{n+7}{3}\right\rfloor$ and $n_{2}=\left\lfloor\frac{n+4}{3}\right\rfloor$.

Proof Let us study the expression

$$
(n+3)\left\lfloor\frac{n+4}{3}\right\rfloor^{2}-2\left\lfloor\frac{n+4}{3}\right\rfloor^{3}+2\left\lfloor\frac{n+4}{3}\right\rfloor+1
$$

depending on the values of $n$. Thus,

- If $n+4=3 p,(8)$ is transformed into $(3 p-1) p^{2}-2 p^{3}+2 p+1=p^{3}-p^{2}+2 p+1$.

- If $n+4=3 p-1,(8)$ is $(3 p-2)(p-1)^{2}-2(p-1)^{3}+2(p-1)+1=$ $p^{3}-2 p^{2}+3 p-1$.

- If $n+4=3 p+1$, (8) is equal to $(3 p) p^{2}-2 p^{3}+2 p+1=p^{3}+2 p+1$. 


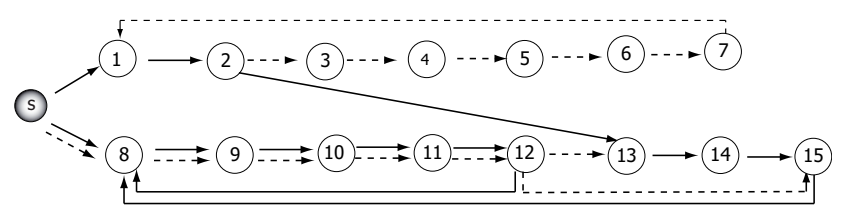

Fig. 10 Digraph as regards to the system examined in example 3.

From above items, their maximum value is $p^{3}+2 p+1$, independently of $p$ whenever $p>0$. In this event, $p=\frac{(n+3)}{3}$ and by substitution, we obtain the inequality given in (7).

\begin{tabular}{|c|c|c|c|c|c|c|c|c|}
\hline$n=n_{1}+n_{2}+k$ & 10 & 15 & 17 & 20 & 25 & 32 & 35 & 36 \\
\hline$n_{1}$ & 5 & 7 & 8 & 9 & 10 & 13 & 14 & 14 \\
$n_{2}$ & 4 & 6 & 7 & 8 & 9 & 12 & 13 & 13 \\
\hline$I_{L R}$ for $\mathcal{F}$ & 89 & 229 & 309 & 465 & 829 & 1609 & 2055 & 2224 \\
\hline
\end{tabular}

In the following example, let us illustrate a positive 2-D systems of order $n=15$ whose $I_{L R}$ is exactly the maximum possible value according to Proposition 1.

Example 3 The positive 2-D system $\left(A_{1}, A_{2}, B_{1}, B_{2}\right)$ of order $n=15$ described by 2-D influence digraph given in Fig. 10 and with a composition table as displayed in Fig. 11 has a locally reachable index $I_{L R}=229$, which is achieved for $n_{1}=\left\lfloor\frac{21}{3}\right\rfloor=7$, and $n_{2}=\left\lfloor\frac{19}{3}\right\rfloor=6$.

\section{Conclusion}

The analysis of local reachability index for positive two-dimensional systems described by the Fornasini-Marchesini state-space model is a complex task from the mathematical point of view. Different quadratic upper bounds on $I_{L R}$ have been consecutively derived in the literature (see [1] and the references therein, [9] and [28]), but being at most $\left(n+3(\lfloor n / 2\rfloor)^{2}\right.$ in the greatest event (see [2]). In this contribution, using the composition table introduced in [2] and limiting the set of entries on the table where a vertex can be deterministically reached, a new family of systems is constructed whose local reachability index can be cubic under suitable conditions, and largely exceeds the aforementioned upper bounds. These results does not clarify the conjectures on $I_{L R}=17$ and $I_{L R}=34$ posed in [2], since they are stated for $n=5$ and $n=7$, respectively, but pointing out once again the mathematical difficulty of the general problem, which is still an open problem. However, the authors are firmly convinced that the composition table can be used to solve the study of an upper bound on the whole, by means of the optimal choice of new influence digraphs that define broad regions of cells in the composition table completely filled with vertices, whose solution is closer to be obtained. 

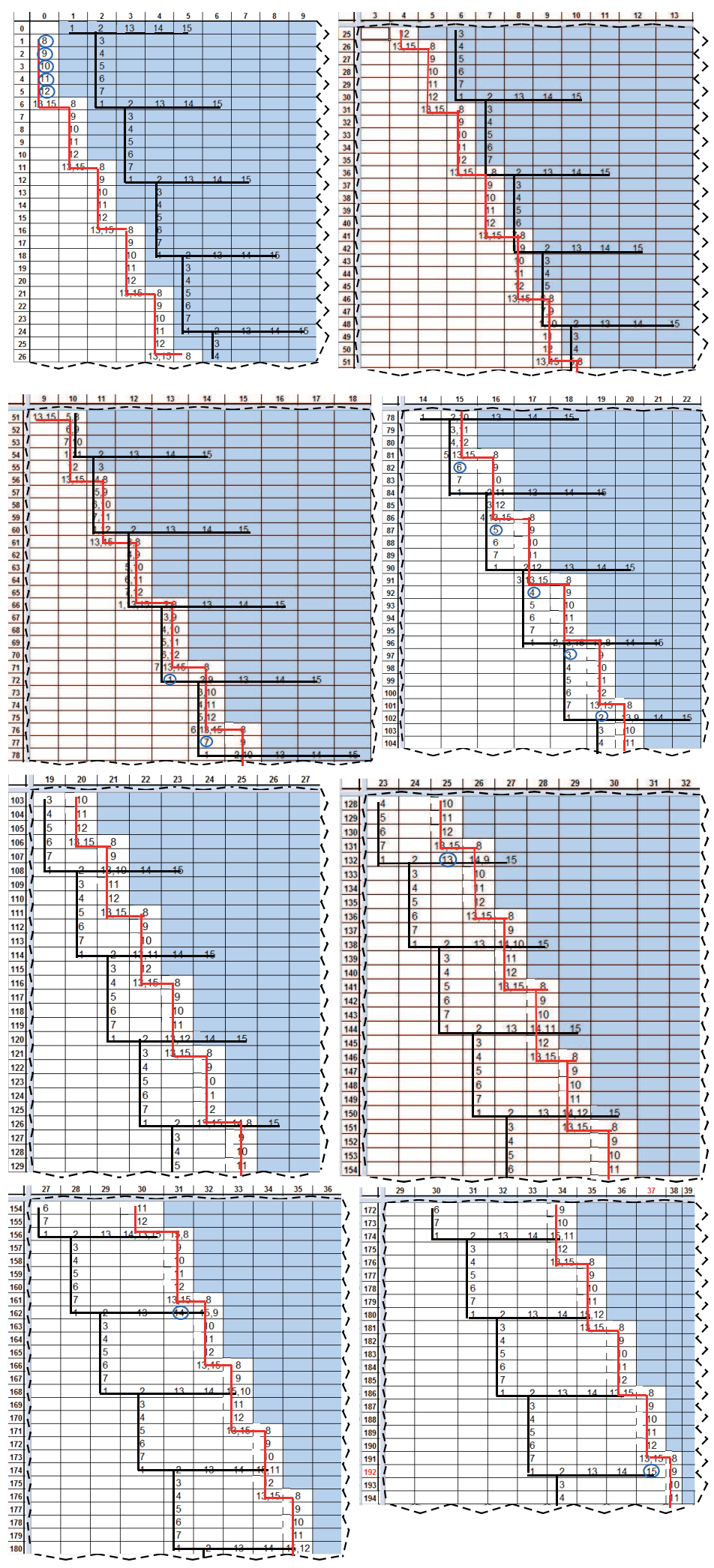

Fig. 11 Composition table associated with example 3. 
Acknowledgements We are gratefully thankful to the reviewers for their valuable remarks. This work has been partially supported by the European Union [FEDER funds] and Ministerio de Ciencia e Innovación through Grants MTM-2013-43678-P and DPI201678831-C2-1-R.

\section{References}

1. E. Bailo, J. Gelonch and S. Romero-Vivo. An Upper Bound on the Reachability Index for a special class of Positive 2-D Systems. Electronic Journal of Linear Algebra, 18:1-12, 2009 .

2. E. Bailo, J. Gelonch and S. Romero-Vivo. Advances on the reachability index of positive 2-D systems. IEEE Transactions on Automatic Control, 59(8): 2248-2251, 2014.

3. Z. Bartosiewicz. Reachability and observability graphs for linear positive systems on time scales. In: IFAC Proceedings Volumes, 47(3), 3803-3808 (2014).

4. L. Benvenuti, A. De Santis and L. Farina (Eds.) Positive Systems: Theory and Applications. In: Lecture Notes in Control and Information Sciences, 294, Springer, Berlin, 2003

5. L. Benvenuti. On the reachable set for third-order linear discrete-time systems with positive control. System and Control Letters, 60(9):690-698, 2011.

6. L. Benvenuti. On the reachable set for third-order linear discrete-time systems with positive control: The case of complex eigenvalues. System and Control Letters, 60(9):10001008, 2011.

7. A. Benzaouia, A. Hmamed and F. Tadeo. Two-dimensional systems: From introduction to state of the art. In Studies in systems, decision and control (Vol. 28). Switzerland: Springer International Publishing. doi: 10.1007/978-3-319-20116-0, 2016.

8. R. Bru and S. Romero-Vivo (Eds.) Positive Systems: Theory and Applications. In: Lecture Notes in Control and Information Sciences, 389, Springer, Berlin, 2009.

9. R. Bru and E. Bailo and J. Gelonch and S. Romero. On the Reachability Index of Positive 2-D Systems. IEEE Trans. Circuits Syst. II: Express Brief, 53(10):997-1001, 2006

10. R. Bru, C. Coll, S. Romero and E. Sánchez. Reachability Indices of Positive Linear Systems. Electronic Journal of Linear Algebra, 11:88-102, 2004.

11. R. Bru, S. Romero-Vivó and E. Sánchez. Reachability indices of periodic positive systems via positive shift-similarity. Linear Algebra and its Applications, 429:1288-1301, 2008 .

12. R. Bru, L. Cacceta and V. G. Rumchev. Monomial Subdigraphs of Reachable and Controllable positive discrete-time Systems. Int. J. Appl. Math. Comput. Sci., 15(1):159$166,2005$.

13. R. Bru and S. Romero and E. Sánchez. Canonical forms of reachability and controllability of positive discrete-time control systems. Linear Algebra and its Applications 310:49-71, 2000.

14. F. Cacace, L. Farina, R. Setola and A. Germani (Eds.). Positive Systems: Theory and Applications. In: Lecture Notes in Control and Information Sciences, 471, Springer International Publishing, Berlin, 2017.

15. B. Cantó, C. Coll and E. Sánchez. On stability and reachability of perturbed positive systems. Advances in Difference Equations, 2014(1): 296, 2014. https://doi.org/10.1186/1687-1847-2014-296

16. C. Coll, M. Fullana and E. Sánchez. Reachability and observability indices of a discretetime periodic descriptor system. Applied Mathematics and Computation, 153:485-496, 2004.

17. C. Commault. A simple graph theoretic characterization of reachability for positive linear systems. Systems and Control Letters, 52(3-4):275-282, 2004.

18. C. Commault. On the reachability in any fixed time for positive continuous-time linear systems. Systems and Control Letters, 56 (4): 272 - 276, 2007.

19. C. Commault and N. Marchand (Eds.) Positive Systems: Theory and Applications. In: Lecture Notes in Control and Information Sciences, 341, Springer, Berlin, 2006.

20. P. G. Coxson and H. Shapiro. Positive reachability and controllability of positive systems. Linear Algebra and its Applications, 94:35-53, 1987. 
21. P. G. Coxson and L. C. Larson and H. Schneider. Monomial patterns in the sequence $A^{k} b$. Linear Algebra and its Applications, 94:89-101, 1987.

22. M. De la Sen. On the reachability and controllability of positive linear time-invariant dynamic systems with internal and external incommensurate point delays. The Rocky Mountain Journal of Mathematics, 40(1):177-207, 2010.

23. M. P. Fanti, B. Maione, and B. Turchiano. Controllability of linear single-input positive discrete time systems. International Journal of Control, 50:2523-2542, 1989.

24. M. P. Fanti, B. Maione, and B. Turchiano. Controllability of multi-input positive discrete time systems. International Journal of Control, 51:1295-1308, 1990.

25. L. Farina and S. Rinaldi Positive Linear Systems: Theory and Applications. Pure and Applied Mathematics, Wiley-Interscience, New York, 2000.

26. E. Fornasini and G. Marchesini. State-Space realization of two-dimensional filters. IEEE Transactions on Automatic Control, AC-21(4):484-491, 1976.

27. E. Fornasini and G. Marchesini. Doubly indexed dynamical systems. Math. Sys. Theory, 12:59-72, 1978.

28. E. Fornasini and M. E. Valcher. On the positive reachability of $2 D$ positive systems. In: L. Farina L. Benvenuti, A. De Santis, editor, Positive Systems, pages 297-304. Lecture Notes in Control and Information Sciences, 2003.

29. E. Fornasini and M. E. Valcher. Controllability and Reachability of 2-D Positive Systems: A Graph Theoretic Approach. IEEE Trans. Circuits Syst. I: Regular Papers, 52(3):576-585, 2005.

30. K. Hrynlów and K. A. Markowski. Experimental evaluation of upper bounds of reachability index for set of solutions of 2-D positive system. 17th International Carpathian Control Conference (ICCC), Tatranska Lomnica, pp. 248-252, 2016, doi: 10.1109/CarpathianCC.2016.7501103

31. T. Kaczorek. Reachability and controllability of $2 \mathrm{D}$ positive linear systems with state feedback. Control and Cybernetics, 29(1):141-151, 2000.

32. T. Kaczorek. Positive $1 D$ and 2D Systems. Springer, London, United Kingdom, 2002.

33. T. Kaczorek. Reachability and minimum energy control of positive 2D systems with delays. Control and Cybernetics, 34(2):411-423, 2005.

34. T. Kaczorek. New reachability and observability tests for positive linear discrete-time systems. Bull. Polish Acad. Sci., Tech. Sci., 55(1):19-21, 2007.

35. T. Kaczorek. Reachability of linear hybrid systems described by the general model. Journal Archives of Control Sciences, 20(2): 199-207, 2010.

36. T. Kaczorek and K. Borawski. Existence of reachable pairs (A, B) of discretetime linear systems. In: Proceedings of 21st International Conference on Methods and Models in Automation and Robotics (MMAR), IEEE, 702-707, 2016. DOI: 10.1109/MMAR.2016.7575222

37. S. P. Kostova. A PLDS Model of Pollution in Connected Water Reservoirs. In: Benvenuti L., De Santis A., Farina L. (eds) Positive Systems. Lecture Notes in Control and Information Science, vol 294, 257-263, Springer, Berlin, Heidelberg, 2003.

38. W. Marszalek. Two-dimensional state space discrete models for hyperbolic partial differential equations Appl. Math. Model., 8(1):11-14, 1984.

39. K. A. Markowski, Determination reachability index space of positive two-dimensional linear system using digraph-based theory 19th International Conference on System Theory, Control and Computing (ICSTCC), Cheile Gradistei, DOI: 10.1109/ICSTCC.2015.7321348, 533-538, 2015. doi: 10.1109/ICSTCC.2015.7321348

40. L. Moysis and V. Mishra. Existence of Reachable and Observable Triples of Linear Discrete-Time Descriptor Systems. Circuits, Systems, and Signal Processing, 1-13, 2018. https://doi.org/10.1007/s00034-018-0922-5

41. R. Pereira, P. Rocha and R. Simões. Characterizations of global reachability of 2D structured systems. Multidimensional Systems and Signal Processing, DOI: 10.1007/s11045011-0154-3, 1-14, 2011

42. V. G. Rumchev and D. J. G. James. Reachability and controllability of time-variant discrete-time positive linear systems, Control and Cybernetics, 33 (1):87-93, 2004.

43. V. Rumchev and S. Chotijah. The Minimum Energy Problem for Positive DiscreteTime Linear Systems with Fixed Final State. In R. Bru, S. Romero-Vivo (Eds.), Positive Systems. Lecture Notes in Control and Information Sciences, 389:141-149, 2009. 
44. M.E. Valcher. Controllability and reachability criteria for discrete time positive systems. International Journal of Control, 65(3):511-536, 1996.

45. M.E. Valcher. Reachability Properties of Continuous-Time Positive Systems. IEEE Transactions on Automatic Control, 54 (7):1586-1590, 2009.

46. M.E. Valcher. Reachability Analysis for Different Classes of Positive Systems. In: R. Bru, S. Romero-Vivo (Eds.), Positive Systems. Lecture Notes in Control and Information Sciences, 389:29-41, 2009. 